\title{
Sens dessus dessous : saint Michel et son Mont
}

Étude à partir d'une enluminure inédite d'un manuscrit breton

\section{Esther Dehoux}

\section{(2) OpenEdition \\ 1 Journals}

\section{Édition électronique}

URL : http://journals.openedition.org/abpo/1425

DOI : $10.4000 / a b p o .1425$

ISBN : 978-2-7535-1490-4

ISSN : 2108-6443

\section{Éditeur}

Presses universitaires de Rennes

\section{Édition imprimée}

Date de publication : 20 juillet 2003

Pagination : $189-208$

ISBN : 978-2-86847-874-0

ISSN : 0399-0826

\section{Référence électronique}

Esther Dehoux, "Sens dessus dessous : saint Michel et son Mont», Annales de Bretagne et des Pays de I'Ouest [En ligne], 110-2 | 2003, mis en ligne le 20 juillet 2005, consulté le 21 avril 2019. URL : http:// journals.openedition.org/abpo/1425; DOI : 10.4000/abpo.1425 


\title{
Sens dessus dessous : saint Michel et son Mont \\ Étude à partir d'une enluminure inédite d'un manuscrit breton
}

\author{
Esther DEHOUX \\ Collège Christine-de-Pisan - Aulnay-sous-Bois \\ Doctorante, université de Limoges
}

En 1998, Sophie Bourdon répertoriait quinze représentations iconographiques du Mont-Saint-Michel réalisées au Moyen Âge ${ }^{1}$, parmi lesquelles six enluminures associaient le mont normand et l'archange. Celles-ci sont conservées dans des manuscrits plus ou moins connus : Très Riches Heures du duc Jean de Berry ${ }^{2}$, Heures du Maréchal de Boucicaut ${ }^{3}$, Heures dites de Sobieski à l'usage de Paris ${ }^{4}$, Heures à l'usage de Nantes ${ }^{5}$, Heures du duc Pierre II de Bretagne ${ }^{6}$, Heures de Louis de Laval ${ }^{7}$.

S'ajoute à ce corpus, l'enluminure des Heures de Montauban ${ }^{8}$ acquises par la bibliothèque municipale de Rennes en 2001.

Il s'agira ici de prendre ces images et de les aborder dans une optique différente : étude d'un lieu saint dans son espace mais aussi étude du double lien unissant le Mont à l'archange mais également Michel à son sanctuaire dans la mesure où ce saint est un des rares, voire le seul, à être clairement associé à un endroit précis. Dans un premier temps, nous présenterons donc ces diverses enluminures puis proposerons des hypothèses d'interprétation.

1. BouRDON, Sophie, Les représentations iconographiques du Mont-Saint-Michel au Moyen Âge, Mémoire de DEA d'histoire de l'art, Université Rennes 2 - Haute-Bretagne, 1998. Ce mémoire est en partie repris et poursuivi dans BOURDON, Sophie, "Quelques représentations médiévales inédites du Mont-Saint-Michel ", Annales de Bretagne et des Pays de l'Ouest, t. 106, n 2, 1999, p. 9-40.

2. Musée Condé, Chantilly, $\mathrm{f}^{\circ} 195$.

3. Musée Jacquemart-André, Paris, $\mathrm{ms} 2, \mathrm{f}^{\circ} 11 \mathrm{v}^{\circ}$.

4. Windsor, The Royal Library, ms $1142 \mathrm{c}, \mathrm{f}^{\circ} 204 \mathrm{v}^{\circ}$.

5. Bodleian Library, ms Add. A 185, $\mathrm{f}^{\circ} 58$.

6. BNF, ms lat. $1159, \mathrm{f}^{\circ} 160 \mathrm{v}^{\circ}$.

7. BNF, ms lat. $920, \mathrm{f}^{\circ} 254 \mathrm{v}^{\circ}$.

8. Bibliothèque municipale de Rennes, $\mathrm{ms} 1834, \mathrm{f}^{\circ} 121$. 


\section{Présentation des manuscrits et description des enluminures}

\section{Heures de Montauban}

Inconnu du public jusqu'à son acquisition par la bibliothèque municipale de Rennes lors de la vente du 10 octobre 2001, ce manuscrit de deux cent huit millimètres sur cent cinquante comporte cent vingt-neuf folios et plus de cent dix miniatures dont trente-sept grandes. Il était destiné à Jean de Montauban dont les armes - champ de gueules à neuf mâcles d'or apparaissent à de multiples reprises dans les enluminures. Né en 1412, Jean, chambellan de Charles VII et maréchal de Bretagne, fait avec le duc François I ${ }^{\mathrm{er}}$, la campagne de Normandie en 1450 et celle de Guyenne sous Charles VII. Il est nommé Grand Maître des Eaux et Forêts et amiral de France en 1461 par le roi Louis XI et meurt en 1466. Réalisé dans les années 1430-1440, ce livre d'heures est l'œuvre du Maître des Heures de Montauban $^{9}$ : les grandes peintures, leur originalité, leur composition, les couleurs sombres et violentes, le cloisonnement sont caractéristiques de cet enlumineur breton du $\mathrm{Xv}^{\mathrm{e}}$ siècle ou, du moins, de son atelier. Ce manuscrit plutôt masculin aurait été la propriété de Jean de Montauban qui aurait pu ensuite l'offrir à sa sœur : le nom d'Isabeau apparaît en effet avec celui de son époux dans le calendrier. Cependant, le calendrier semble avoir été rajouté après et aucun document d'archive n'a permis, à ce jour, d'apporter plus de précision sur l'histoire de ce manuscrit et sur des dons ou legs éventuels dont il aurait pu faire partie.

Le folio 121 est consacré à saint Michel : dans un cadre doré portant, en rouge et bleu, la première phrase du répons de la leçon VIII de l'office du 29 septembre "Michael archangele veni in adjutorium in populo Dei in angelorum conspectu ", se distinguent neuf ensembles : en haut, au centre une représentation du Mont-Saint-Michel, à droite une intervention salvatrice de l'archange. Au centre, une zone de texte relative à l'archange placée au-dessus d'une représentation de Michel pesant les âmes lors du Jugement dernier. À gauche de cette dernière le Paradis et à droite l'Enfer. Les autres cadres sont des décors floraux classiques dans leur forme et leurs couleurs.

Le Mont est représenté de façon originale car plusieurs angles de vue sont mêlés. La mer est haute et porte quatre navires dont trois paraissent être de taille imposante par rapport à la barque dans laquelle un homme semble sommeiller. On distingue en haut des mâts des oriflammes rouges rappelant la tenue du personnage endormi. En effet, celui-ci porte un vêtement rouge et jaune qui pourrait être une livrée aux couleurs de son mấtre. À l'extérieur de l'enceinte, le rocher est abrupt, découpé et recouvert

9. BNF, ms lat. 18026. Ce manuscrit présente de très grandes similitudes avec celui de la Bibliothèque municipale de Rennes mais il semblerait qu'il aurait appartenu davantage à l'épouse de Jean de Montauban qu'au seigneur de Montauban lui-même : en effet, de nombreuses enluminures et notamment celle du commanditaire représentent une femme. 
d'une végétation rase mais verdoyante. Le village, composé d'une dizaine de maisons aux toits noirs, est protégé par un rempart crénelé muni d'une porte avec une herse, de tours circulaires à la couverture orangée et garni de meurtrières. Une passerelle de bois permet d'accéder à l'abbaye en passant le mur équipé de créneaux et de meurtrières qui la sépare du village. L'église abbatiale, dépassant légèrement du cadre, est figurée de côté avec deux niveaux d'ouvertures, six clochetons dont deux dotés de toitures orangées et le clocher central dominant la toiture noire. Éléments frappants de cette représentation : les croix qui ornent tous les bâtiments et les fleurs de lis dorées sur fond bleu présentes sur l'abbatiale sous la forme d'oriflammes et, sous la forme d'écu, sur les remparts ainsi qu'aux extrémités supérieures du cadre. Dans le ciel bleu se détachent, à gauche, une colombe rayonnante et, à droite, semblant sortir d'une des tours, le visage paisible, Michel. Vêtu de blanc, auréolé, aux ailes dorées ocellées, il fond sur un diable qu'il agrippe par la patte arrière gauche et va vraisemblablement frapper avec l'épée qu'il brandit de la main droite. Le diable noir possède un corps étrange : des membres allongés munis de griffes, un museau fin et allongé d'où sort une langue fourchue, des oreilles longues et pointues, de courtes ailes et une imposante queue touffue.

Dans le cadre supérieur droit, Michel, toujours auréolé et aussi serein, est revêtu d'une cuirasse bleue et de protections noires articulées pour les membres. Il porte un manteau doré semé de bleu, doublé de rouge et rejeté sur l'épaule se détachant bien des ailes amarante à l'extérieur et bleues parsemées de plumes vertes et jaunes à l'intérieur. Tout son corps et ses vêtements rayonnent. Il brandit de la main droite une lance crucifère qu'il plante avec facilité dans le bas-ventre d'un diable noir renversé en partie coupé par le bord de l'enluminure. Celui-ci est blessé : du sang s'écoule. L'intervention de l'archange permet la délivrance de l'humain représenté nu qui semble s'agripper aux ailes de Michel pour échapper aux griffes diaboliques. La scène se déroule dans un milieu rappelant le Mont : même ciel bleu, même sol verdoyant découpé, une bande grise comparable à la mer sépare cet espace d'un îlot sur lequel on distingue, à gauche, des constructions fortifiées ressemblant à celles du rocher normand.

Les trois cadres inférieurs sont liés et relatifs au Jugement dernier. Dans la partie droite, dans un décor architectural - on distingue la voûte verte, les piliers dorés et le mur gris percé de baies - Michel toujours auréolé et muni de ses protections noires, a revêtu une tunique rouge et tient une épée dans la main droite. De la main gauche, il tient, par la barbe, un diable noir velu tenant une âme pour la conduire en enfer. La position de cet être diabolique couvert de longs poils noirs et muni de griffes acérées est étrange : le bas du corps tourne le dos à l'archange, le torse est de face alors que la tête regarde Michel. Sa tête anguleuse possède de grandes oreilles pointues, une large bouche s'ouvrant sur des dents effilées, des cornes et une chevelure hirsute. En bas de ce combat : deux diables plus petits mais présentant les mêmes caractéristiques transportent sur leur dos ce qui semble être des âmes destinées à l'enfer. L'importance de leur 
charge leur fait plier l'échine. On retrouve un diable semblable sous le bras droit du grand diable dans le cadre central. Dans celui-ci, la pesée des âmes : au centre, Michel tenant la balance dans sa main gauche. Il porte la même tenue que dans la représentation en haut à droite : protections noires, tunique bleue, manteau rouge et or et auréole. On notera seulement ici que les articulations de l'armure apparaissent en doré. Dans sa main gauche, il tient une lance crucifère qu'il appuie avec force dans le plateau droit de la balance. Le mouvement général du corps tend à montrer la fermeté de la volonté et du geste : il s'agit de sauver l'âme en prière de l'enfer et de peser plus fort que le diable qui appuie ses pattes griffues sur les épaules de l'humain mauvais représenté la bouche ouverte, la langue tirée, tenant dans les mains un objet jaune difficilement identifiable. L'issue de jugement semble favorable : l'âme échappera à l'enfer. Représentée à gauche de l'archange, l'entrée de l'enfer est symbolisée par une immense gueule noire béante dotée de quatre dents énormes. À l'intérieur : un diable noir cornu aux incisives proéminentes représenté comme le prince des lieux : il porte une couronne fleuronnée mais est représenté de profil, en fausse majesté. L'environnement rouge laisse voir, à l'arrière-plan, les réalités infernales : on distingue cinq âmes cuisant dans une marmite posée sur un feu aux vives flammes rouges et, au fond, cinq damnés pendus à une potence. Ces dix malheureux voués à l'enfer semblent regarder les élus, bien plus nombreux, menés par une femme vêtue d'une robe rouge écarlate au col doré vers le Paradis. La scène se poursuit dans le cadre gauche : sous un ciel bleu sombre étoilé, les âmes sauvées sont conduites par deux anges portant chacun une croix rouge semblable à celle de Michel, celui de gauche aux ailes bleues est vêtu d'une longue tunique blanche tandis que celui de droite, vêtu d'amarante, porte des ailes dorées. Ils montent les marches menant à un bâtiment d'architecture riche : clochetons multiples, voûte d'ogives rouge comme la toiture, gâble ajouré surmonté d'un pinacle doré sur lequel flotte l'étendard de Jean de Montauban, piliers dorés également. Cet édifice est très certainement une représentation du Paradis, de la Jérusalem céleste fréquemment imaginée sous la forme d'une église par les artistes ${ }^{10}$.

\section{Heures du Maréchal de Boucicaut}

Volume de deux cent quarante-deux folios de deux cent soixante-quatorze millimètres sur cent quatre-vingt-dix, ce manuscrit fut réalisé entre 1405 et 1408 pour Jean Le Meingre (1365-1421), dit Boucicaut, comte de Beaufort, vicomte de Turenne, maréchal de France, par un artiste anonyme désigné " Maître de Boucicaut ".

L'enluminure consacrée au mont normand est au folio $11 \mathrm{v}^{\circ}$ : c'est une représentation en pleine page, sans texte, entourée d'un cadre doré et soulignée par une fine bande très sobrement décorée de motifs floraux dorés,

10. Delumeau, Jean, Que reste-t-il du Paradis?, Paris, 2000, p. 99. 
rouges et bleus. Dans la partie supérieure de l'image : un paysage marin où émergent deux rochers : le Mont-Saint-Michel et, plus en retrait, le rocher de Tombelaine ou les hauteurs d'Avranches. Sur la mer légèrement agitée, quelques oiseaux dont des cygnes, et un bateau. Dans la partie inférieure de l'image : un paysage rocailleux parsemé d'arbustes servant de cadre au combat archangélique auquel l'enlumineur a consacré les trois quarts de la page. Dominant l'ensemble : un grand soleil doré rayonne dans un ciel bleu étoilé.

Le Mont est représenté de façon originale car vu de l'est ${ }^{11}$ : le village n'apparaît qu'à l'extrême gauche en arrière d'un mur défensif garni de tours. En dehors de cette zone habitée : un espace pentu, rocailleux à gauche et recouvert ailleurs d'une végétation assez rase dans l'ensemble. À l'intérieur de l'enceinte, on distingue quatre pignons de maisons aux toits orangés dans la partie basse et deux autres dans la partie haute, proche de l'abbaye. Derrière le rempart grisé, les bâtiments religieux représentés en rosé avec les toits bleus et le clocher en flèche caractéristique de l'abbatiale. Malgré un certain réalisme, l'image semble davantage un assemblage de vues plus qu'une vue du Mont-Saint-Michel.

L'archange est le personnage central : au milieu d'un paysage rocailleux parsemé d'arbres souvent de petite taille, il domine un démon. Protégé par une armure articulée dorée - comme ses ailes - recouverte d'un riche manteau rouge doublé de bleu et garni d'un orfroi doré, il tourne sa tête auréolée et couronnée d'une croix vers son adversaire vaincu. Michel domine sans conteste son ennemi : son pied droit posé sur le genou gauche, l'autre sur le thorax, il appuie, de sa main gauche, sur le cou du vaincu, son écu doré marqué d'une croix rouge et, de l'autre main, plante sa pique crucifère dans la gorge. Le démon est couché sur le sol : c'est un être vilain de couleur marron, ailé, velu, muni de longues cornes et de griffes acérées. Il tente de résister : les mouvements désordonnés de ses membres et de sa tête, la crispation de l'ensemble traduisent sa volonté de ne pas s'incliner tout comme son bras droit repoussant le bouclier angélique. La violence et la haine qu'exprime son visage aux traits grossiers contrastent avec la sérénité michaëlienne.

\section{Très Riches Heures du duc de Berry}

Volume de deux cent soixante-six folios de deux cent quatre-vingt-dix millimètres sur deux cent dix en vélin très fin, ce manuscrit compte soixante-six grandes miniatures et soixante-cinq petites et date des années 1410-1416. Les auteurs des enluminures sont Paul, Herman et Jean de Limbourg, originaires de Nimègue, qui travaillèrent pour Philippe le Hardi avant de servir Jean de Berry, destinataire de ce livre d'heures. Disparus de façon inexpliquée en 1416, année de la mort du duc, ils laissent le manus-

11. BouRDON, Sophie, Les représentations iconographiques..., op. cit., p. 106-109. 
crit inachevé. Entre 1485 et 1489, l'enlumineur berruyer Jean Colombe le complète ${ }^{12}$.

L'enluminure qui nous intéresse ici est celle du folio 195 : elle met en scène en pleine page, pour le 29 septembre, date de la Saint-Michel, le MontSaint-Michel avec, au-dessus du monument, dans un ciel bleu profond, l'archange combattant le dragon. Autour du thème central, sept médaillons entourés de nuages rayonnant représentant des anges très attentifs au déroulement de la lutte. Exception cependant : l'ange portant l'écu du duc de Berry, dans le médaillon situé en bas, à gauche, regarde ailleurs.

Le Mont est représenté avec minutie, réalisme ${ }^{13}$, vu du côté sud, à marée basse. En contrebas, la grève sur laquelle sont échouées cinq embarcations, le village de maisons à pans de bois et aux toits de tuiles rouges avec son enceinte et son église paroissiale nettement différenciée des habitations par son toit bleu. Plus haut, entre le village et l'abbaye, le rocher inhabité, couvert d'une végétation très rase. Au-delà du mur qui l'isole du monde, l'abbaye est dessinée en gris et bleu avec ses diverses composantes et notamment l'église abbatiale dominant la totalité du rocher.

Dominant le Mont, l'archange protégé par une cuirasse et une cotte de mailles bleues, vêtu d'une longue tunique bleue également, est représenté avec les membres, la tête, l'auréole et l'extrémité des ailes rouges et dorés. Il tient dans sa main gauche un fourreau duquel il a tiré l'épée d'estoc qu'il brandit de la main droite. Il vient d'en asséner un coup au dragon. Corps vert écaillé se terminant par une longue queue s'enroulant sur elle-même, ailes de chauve-souris déployées, courtes pattes aux griffes acérées, cou longiligne, tête fine avec une large gueule ouverte d'où sort une langue rouge fourchue : le dragon est blessé au cou car du sang s'écoule. L'attitude des deux protagonistes ne laisse pas de doute quant à l'issue du combat : le corps dynamique en extension totale de l'archange s'oppose à celui du dragon replié sur lui-même et tourné vers le sol.

\section{Heures dites de Sobieski}

Daté de 1420 et enluminé par le Maître de Bedford, le manuscrit des Heures dites de Sobieski présente une illustration en pleine page du mont normand au folio $204 \mathrm{v}^{\circ}$. La grande précision du dessin, la forte vraisemblance sont comparables à celles de l'enluminure des frères Limbourg mais l'organisation de la représentation et la technique sont très différentes. Au tiers de la hauteur du feuillet, un rectangle de texte occupe les deux tiers de la largeur de la page et sépare le bas de l'image de la partie supérieure où est représenté le sanctuaire normand. Dans le bas de l'image, à droite,

12. Champeaux, A. de, "Jean Colombe enlumineur des ducs de Savoie et de la reine de France Charlotte de Savoie ", Chronique des arts et de la curiosité, 1895, p. 154-155.

13. BouRdon, Sophie, Les représentations iconographiques..., op. cit., p. 82 à 90 . Elle note, page 90, que " cette description représente un des plus importants témoignages iconographiques des anciennes structures du monument". 
une auberge que quitte un groupe de pèlerins se rendant, à pieds ou en chariot tiré par un cheval, au Mont. Ces pèlerins, identifiables à leurs sacoches et à leurs bourdons, sont de conditions sociales différentes : les trois plus à droite semblent être issus de milieux plus modestes que les trois personnages sur le chariot. La précision dans le rendu des individus et notamment de leurs vêtements permet de distinguer les tenues longues et riches de ces derniers qui s'opposent aux habits plus courts, plus sobres des premiers ${ }^{14}$. Entre les deux groupes, un clerc portant la robe et le capuchon noir. À gauche : des navires et, au dessus et en dessous de ceux-ci, deux représentations de Michel combattant le démon.

Le Mont est vu du sud et présente de façon très nette, derrière le rempart, le village, l'abbaye avec son système défensif et l'église abbatiale. L'impression de hauteur est assez peu rendue comme en témoigne l'espace séparant le village de l'abbaye. Elle est suggérée par les escaliers que gravissent les pèlerins. On en dénombre quatre dans l'enceinte du village : deux, près de la porte, munis de bourdons, un troisième grimpant les marches du porche et le dernier, de taille disproportionnée, gravissant les derniers degrés conduisant à l'abbaye. On note la présence d'un chien dans le bas du village. La précision du dessin permet d'identifier certains bâtiments du sanctuaire ${ }^{15}$, toujours dominé par l'église abbatiale dont la flèche se termine, hors du cadre, par une croix.

Michel est représenté à deux reprises et, à chaque fois, il combat un démon dans les airs pour permettre aux pèlerins d'accéder au sanctuaire. Dans la scène en haut à gauche, l'archange, la tête rayonnante, protégé par une armure rigide articulée et un écu croisé qu'il tient dans sa main gauche, plante sa lance dans le corps du démon. Celui-ci est de petite taille, noir, cornu, griffu et ailé : il tente de se défendre de la main gauche tandis que, de sa main, droite, il tient le mât du navire qu'il veut détourner du rocher. Ce bateau est occupé, semble-t-il, par un second démon comparable au premier, qui met bas les voiles pour anéantir tout espoir de parvenir au Mont. En bas, Michel vole littéralement au secours des pèlerins qui semblent, eux, ignorer qu'ils sont agressés par un envoyé du Diable. Celuici, identique aux deux premiers, plante sa fourche dans le chariot menant les fidèles à l'abbaye. L'archange vêtu d'une tunique blanche et d'une cape, attaque l'être démoniaque avec son épée dans la main droite et son bouclier dans la gauche.

\section{Heures à l'usage de Nantes}

Conservé à la Bodleian Library, ce manuscrit français est daté de 1435 par König ${ }^{16}$ mais certaines études plus récentes tendraient davantage à le

14. Blanc, O., Parades et parures. L'invention du corps de mode à la fin du Moyen Âge, Paris, 1997.

15. BouRDON, Sophie, Les représentations iconographiques..., op. cit., p. 114-119.

16. KonIG, E., Französische Buchmalerei um 1450, Berlin, 1982, p. 136-140; 152; 184-188; 203-204. 
dater des années 1450-1455 ${ }^{17}$. Il compte quarante et une peintures insérées dans le texte.

Au folio 58, consacré à l'archange, l'enluminure, de forme carrée, placée en haut à gauche, entourée de marges classiquement décorées de feuilles d'acanthe notamment, représente Michel combattant un diable avec, en arrière plan, se détachant dans un ciel en grande partie blanc, le Mont-Saint-Michel. Le milieu immédiat de la lutte est sobre : à gauche du saint, un rocher abrupt et sur le reste de la largeur, une étendue uniformément verte avec quelques petits arbustes près du démon et au bord de la mer. À l'horizon, au-delà de la mer : à gauche, quatre pèlerins se dirigeant vers le Mont-Saint-Michel représenté à droite. Dans la partie inférieure du rocher, le village apparemment non fortifié et, au sommet, une construction protégée par un rempart. Derrière les quatre tours aux toits pointus de l'enceinte, on distingue un pignon blanc et une toiture orangée. La scène doit se passer à marée basse mais l'angle de vue est difficile à définir. Le mont normand manque, par rapport aux autres enluminures, de précision dans le bâti mais il reste cependant bien reconnaissable.

L'archange, exceptionnellement dépourvu d'ailes, porte un manteau rouge à orfroi doré doublé d'hermine sur son armure intégrale articulée bleue. Il piétine avec sérénité un démon vert sombre, décharné, aux membres longs et griffus et doté d'ailes semblables à celles des chauve-souris. Sa tête présente une sorte de bec pointu ouvert, de grandes dents blanches, des incisives proéminentes, de grandes oreilles allongées. L'issue du combat est claire : Michel a planté, de sa main droite, sa pique crucifère dans le thorax de son adversaire qui tente vainement de résister : de sa main gauche, il saisit la lance pour la repousser et tente d'atteindre l'archange de sa main droite. On notera que Michel écarte son bras gauche du corps et présente un poing serré qui ne tient rien ${ }^{18}$.

\section{Heures de Pierre II de Bretagne}

Réalisé pour le duc Pierre II, ce manuscrit de cent quatre-vingt-dix millimètres sur cent trente daterait donc des années 1450-1457 et plus précisément des années 1455-1457 ${ }^{19}$. Les enluminures sont de qualité moyenne

17. AvRIL, F., ReYnAUD, N., Les manuscrits à peintures en France, 1440-1520, Paris, 1995, p. 132 .

18. BouRDon, Sophie, Les représentations iconographiques..., op. cit., p. 134, n. 170 note « On peut penser que cette main devait à l'origine tenir la lance, ce que ce geste de saisie nous pousse à croire, ainsi que la droite induite par la ligne supérieure des phalanges qui peut être poursuivie jusqu'à atteindre le démon à la gueule. Comment expliquer cette "erreur" sur la miniature définitive, sinon par une correction de l'artiste qui, en cours de réalisation de son œuvre, se rend compte qu'il ne peut raisonnablement faire brandir son arme à l'archange Michel de la main gauche? "

19. Ibid., p. 151 : « La présence, dans les suffrages, de saint Vincent Ferrier au folio $128 \mathrm{v}^{\circ}$ permet de ramener la datation à 1455 , année de la canonisation de ce dernier, et 1457, année de la mort du duc." 
notamment pour la représentation des personnages mais l'ingéniosité des encadrements et l'harmonie des coloris compensent les maladresses.

La miniature du folio $160 \mathrm{v}^{\circ}$ est bien ordonnée : un cadre central représentant la lutte opposant Michel au dragon et, dans l'angle inférieur gauche, un encadré plus petit consacré au Mont-Saint-Michel et aux pèlerins s'y rendant. Sous l'archange, les premiers mots de l'antienne en son honneur : De sancto Michaele archangelo an[tiene]. Michael archangele veni in adjutorium populi in conspectu angelorum. L'ensemble est entouré d'un ruban orné de motifs végétaux. Les marges ont été décorées de manière traditionnelle avec l'emploi de larges feuilles d'acanthe bicolores bleues et dorées ou bleues et brunes ainsi que de fines fleurs bleues et rouges. On note la présence d'un plant de fraisier dans le bas : sortant d'une vasque dorée, il s'épanouit de façon symétrique. Dans l'angle supérieur droit, on observe un paon qui, au-delà de l'aspect purement décoratif, symbolise l'éternité et l'immortalité ${ }^{20}$.

La présence et la superposition des cadres expliquent la forte verticalité du Mont : il est en effet représenté dans l'extrémité gauche et très peu large afin de ne pas être masqué. Cette représentation est une association de deux vues : une du sud pour l'entrée du village et l'autre de l'est pour la rue face à la porte du monastère. Dans la partie inférieure, un fond beige tacheté de brun rappelant la grève et une douzaine de personnages se rendant à pieds, à cheval ou en chariot à l'abbaye dont l'entrée semble gardée par un homme en armes. Munis de bourdons et de sacoches, ce sont des pèlerins : ils portent tous un chapeau et sont vêtus, pour la plupart, d'habits bleus ou rouges. L'extérieur du village et de l'abbaye est abrupt, rocailleux, doté seulement de quelques arbres répartis sur les différents paliers. À l'intérieur du rempart crénelé et doté de tours, deux autres fidèles montent la rue bordée de maisons qui rejoint l'église. Le village est composé d'habitations blanches aux toitures bleutées accolées les unes aux autres mais décalées en hauteur en raison de la pente. L'enceinte de l'abbaye ressemble à celle du village : créneaux, tours angulaires. On distingue, couvertes d'une toiture bleue se détachant dans le ciel blanc, l'église reconnaissable à sa flèche et, à droite, une tour circulaire avec un clocher. Quelques oiseaux volent dans le ciel au-dessus du sanctuaire. La mer, représentée en bleu-vert, sépare le Mont d'un rocher surmonté d'une construction fortifiée qui pourrait être Tombelaine à droite et de la côte habitée à gauche.

Un motif quadrillé de multiples couleurs remplacé aux deux tiers de l'image par une perspective de biais faite d'une sorte de carrelage alternant des carreaux verts et bleus donnant une illusion de sol sert de cadre au combat. Le corps totalement recouvert d'une armure articulée, vêtu d'une cape blanche à l'intérieur rouge, auréolé, les grandes ailes dorées et bleues ocellées déployées, l'archange tient dans sa main droite une épée

20. Coll., Missel pontifical de Michel Guibé, XVe. Cérémonie du couronnement des ducs de Bretagne, Rennes, 2001, p. 83. 
d'estoc avec laquelle il va frapper le démon qu'il tient par l'oreille. L'être diabolique jaune-vert, vilain, décharné, présente des membres longs se terminant par des griffes rouges acérées. Il possède des ailes de chauve-souris, une courte queue pendante, de grandes oreilles écartées, de longues cornes rouges, une large bouche ouverte d'où sort une langue rouge. Ses gestes désordonnés ne masquent pas le fait qu'il est contraint à s'agenouiller par Michel et donc vaincu.

\section{Heures de Louis de Laval ${ }^{21}$}

Commencé vers 1470-1475 et repris entre 1485 et 1489, ce manuscrit d'une grande richesse est une des œuvres les plus remarquables sorties de l'atelier de Jean Colombe. Volume de trois cent quarante-deux folios mesurant deux cent quarante-trois millimètres sur cent soixante-douze, il comporte plus de mille deux cent trente quatre enluminures dont cent cinquante-sept en pleine page. Ce manuscrit était destiné à Louis de Laval, seigneur de Châtillon, gouverneur de Champagne et grand maître des Eaux et Forêts de France représenté à plusieurs reprises ${ }^{22}$ et a été transmis à Anne de France, dame de Beaujeu et duchesse de Bourbon après la mort de son propriétaire en 1489. La partie la plus ancienne de ce livre d'heures est celle allant des folios trente à trois cent quarante deux dont la décoration aurait été faite par le Maître du Missel de Yale et par Colombe et c'est là que se trouve l'enluminure consacrée à l'archange.

Au folio $254 \mathrm{v}^{\circ}$, dans un cadre ocre brun décoré de coquilles titré Michael archangele veni in adjuto, l'artiste a représenté le combat de Michel face au démon en occupant le maximum d'espace. Représenté dans les airs, Michel arbore des ailes blanches et une cape rouge voletant. Ces attributs lui donnent une impression de volume qui contraste avec la finesse de son corps. Revêtu intégralement d'une armure dorée, il semble évoluer avec facilité et sérénité : les jambes légèrement pliées, il plante sa pique crucifère dans l'arrière-gorge du démon vert sombre. Représenté à genoux, celuici fixe d'un regard haineux son vainqueur. Original dans sa forme, il associe des caractéristiques diverses : les extrémités de ses membres sont comparables à celles d'un coq, ses cuisses sont larges et poilues, ses épaules semblent recouvertes de plumes, son corps paraît être écaillé, sa queue est celle d'un lion. Sa tête, légèrement rouge, aux traits grossiers, possède de larges oreilles, de longues cornes tournées vers l'avant, un gros nez épaté et une très large bouche entrouverte. Sa blessure au cou saigne abondamment : deux filets de sang coulent sur sa poitrine et sur le sol.

21. SCHAEFER, C., " Nouvelles observations au sujet des Heures de Louis de Laval ", Arts de l'Ouest, 1980, p. 33-68.

22. BouRdon, Sophie, Les représentations iconographiques..., op. cit., p. 163. Aux folios $50 \mathrm{v}^{\circ}$ et 51 , il est représenté, âgé, en prière devant Marie et l'Enfant. Au folio $334 \mathrm{v}^{\circ}$, il est peint jeune priant devant son tombeau. 
L'arrière-plan est un paysage mêlant collines et eau : à gauche, à mi-hauteur environ, une île fortement boisée, à droite, une première colline très abrupte recouverte d'une végétation assez rase et surmontée d'une construction fortifiée coupée par le bord du cadre, une deuxième avec une verdure abondante dans sa partie inférieure et, au sommet, des tours circulaires, des murs crénelés faisant penser à un château. Au fond, un relief ondulé, légèrement boisé se détachant dans la brume et séparé par un bras de mer d'une colline très pentue, recouverte d'arbres dans sa partie inférieure droite par opposition à la gauche, au sommet de laquelle on distingue, malgré la cape de l'archange, une construction faite de trois tours entourées d'un rempart.

Sophie Bourdon pense que le Mont-Saint-Michel pourrait être la deuxième colline à droite notant qu'elle est " sans doute la plus petite mais également la plus proche, proportionnellement parlant, de la forme très globale du Mont ${ }^{23}$ ". Exposant les limites de l'identification, elle présente deux hypothèses opposées ${ }^{24}$ : la première assimilant ce paysage à un cadre de campagne médiévale avec des mottes castrales et la seconde insistant sur l'originalité de présenter un milieu marin et sur le lien très fort dans la réalité comme dans l'imaginaire entre le mont normand et la mer. Elle semble opter pour la seconde en notant que « l'image doit alors être comprise en tant qu'illustration de la baie, observée depuis l'est où se place le combat du premier plan et regardant vers l'ouest, c'est-à-dire vers la colline la plus éloignée à droite de l'arrière-plan qui figurerait alors le Mont-Dol ${ }^{25}$..." mais elle reconnaît manquer de certitudes : le rocher en question est le plus petit, aucune maison n'est présente sur ses flancs, aucun clocher, aucune croix ou flèche ne vient figurer une présence religieuse; la seule raison qui pourrait plaider en la faveur d'un Mont-Saint-Michel potentiel est la présence de la mer assez rare dans les représentations de l'archange Michel $^{26}$. Il semble cependant étonnant que Jean Colombe, connaissant les Très Riches Heures du duc de Berry pour les avoir achevées, ait si peu tenu compte du modèle qu'il avait en sa possession pour représenter le sanctuaire normand. Les paysages de l'artiste berruyer se caractérisent par des premiers plans souvent abrupts qui contrastent avec des horizons vaporeux généralement peints en bleu, des montagnes ou des collines, au modelé rendu grâce à de petites lignes parallèles, couvertes de châteaux, des étendues aqueuses fréquentes. L'arrière-plan de l'enluminure consacré à l'archange serait donc un environnement souvent peint par Colombe. Sachant, de plus, qu'il a séjourné en Italie - un acte du 3 juin 1486 signé, en sa présence, par Charles I ${ }^{\mathrm{er}}$ à Turin en témoigne - et que l'art italien à la fin du Moyen Âge se caractérise par des arrière-plans associant la terre, la mer, l'air dans une symbolique de l'univers plus que dans une représentation

23. Ibid., p. 168.

24. Ibid., p. 169-170.

25. Ibid., p. 170.

26. Ibid., p. 170-171. 
réaliste du cadre, on pourrait envisager de voir ici un paysage sans identité définie plus qu'un très hypothétique Mont-Saint-Michel et donc d'écarter cette enluminure de notre corpus.

\section{Signification et interprétation}

La lutte contre le dragon est l'illustration de l'Apocalypse :

" Il y eut alors une grande bataille dans le ciel : Michel et ses anges combattaient contre le dragon, et le dragon avec ses anges combattaient contre lui. Mais ceux-ci furent les plus faibles et depuis ce moment ils ne parurent plus dans le ciel. Et ce grand dragon, cet ancien serpent qui est appelé Diable et Satan, qui séduit tout le monde fut précipité en terre et ses anges avec lui ${ }^{27}$. "

Le combat archangélique, très fréquent dans l'iconographie religieuse médiévale, est le signe d'une dévotion importante à l'archange. Dans notre corpus, il est net que ce thème est souvent le sujet principal : les Heures de Boucicaut, les Heures à l'usage de Nantes, les Heures de Pierre II en témoignent. Dans ces trois cas, le Mont-Saint-Michel apparaît comme décor voire dans un cadre en retrait. Cependant, dans le manuscrit rennais, les Très riches Heures du duc de Berry et les Heures dites de Sobieski, le rocher normand occupe une place primordiale : le combat archangélique est représenté soit dans des cadres de taille plus réduite, soit à des places d'importance moindre. On note l'originalité des Heures de Montauban qui sont les seules à associer à ces deux thèmes celui du Jugement dernier. Toutefois, on observe que la disposition d'un thème par rapport à l'autre n'est pas identique d'un manuscrit à l'autre : Michel apparaît tantôt en dessous ${ }^{28}$, tantôt au-dessus ${ }^{29}$ voire à côté ${ }^{30}$, le manuscrit rennais étant le seul à présenter les trois à la fois. Il s'agit là de montrer la protection spéciale dont bénéficie le sanctuaire : elle s'exerce face aux menaces venant des airs, de la terre, des hommes, des démons et autres créatures diaboliques. Dans une telle optique, l'assimilation est rapidement faite : tout ennemi du Mont est ennemi de Dieu.

Comme le dit J.-C. Schmitt, " la construction de l'espace de l'image et l'agencement de figures entre elles ne sont jamais neutres : ils expriment et produisent tout à la fois une classification des valeurs, des hiérarchies, des choix idéologiques ${ }^{31}$ ". Ainsi, formes, couleurs, attitudes participent du réalisme mais sont aussi lourdes de signification pour l'œil médiéval qu'il soit celui du commanditaire, du réalisateur ou du spectateur.

27. Apocalypse de Jean, XII, 7-9.

28. Heures de Montauban, Heures de Boucicaut, Heures à l'usage de Nantes.

29. Heures de Montauban, Très Riches Heures du duc de Berry, Heures dites de Sobieski, Heures de Pierre II de Bretagne, Heures de Laval.

30. Heures de Montauban, Heures dites de Sobieski.

31. Sснмітт, J.-C., Le Corps des images. Essai sur la culture visuelle au Moyen Âge, Paris, 2002, p. 43. 


\section{Conventions et codes}

L'opposition archange/être diabolique, Bien/Mal est le thème principal de ces enluminures, l'archange étant ici le représentant du Bien luttant contre le Mal incarné dans le dragon ou le démon. Très manichéenne, cette distinction s'opère de manière systématique par le biais de divers facteurs obéissant à des normes ${ }^{32}$.

Le physique des personnages traduit cette différence mais c'est surtout la déformation, les exagérations qui ont un pouvoir expressif fort en se démarquant de la configuration commune et banale ${ }^{33}$. L'être malfaisant se définit plus par son animalité que par le fait d'être strictement animal ${ }^{34}$. On observe ainsi souvent chez lui des caractères typiques d'animaux appartenant au bestiaire diabolique plus qu'une identité stricte : queue reptilienne du dragon des Très Riches Heures de Berry, cornes de caprins dans toutes les enluminures à l'exception des heures de Berry, ailes de chauvesouris et griffes acérées comme dans l'ensemble de notre corpus. La tête est, selon Michel Pastoureau, une " armoirie corporelle ${ }^{35}$ " : faciès convulsé des Heures de Boucicaut, des Heures de la Bodleian Library ou des Heures de Pierre II; bouche ouverte, tordue et grimaçante comme dans l'ensemble de notre corpus laissant ainsi parfois sortir la langue en signe de dérision pour les choses sacrées comme dans les Heures de Montauban, les Très Riches Heures - où la langue est, de surcroît, fourchue - les Heures dites de Sobieski et les Heures de Pierre II; lèvres parfois épaisses en signe d'hypocrisie, d'infidélité, de mensonge comme dans les Heures de Pierre II ou celle de Boucicaut; nez proéminent comme dans les Heures de Montauban ou, au contraire, épaté comme dans les Heures de Boucicaut ou de Pierre II; oreilles développées et pointues comme dans le manuscrit rennais, les Heures dites de Sobieski et celles de la Bodleian Library ou écartées comme dans le manuscrit de Boucicaut et celui de Pierre II.

À ces caractéristiques physiques s'ajoute un comportement : l'être diabolique est crispé et présente une gestualité désordonnée. Il s'oppose ainsi point par point à l'être saint qui possède un physique irréprochable avec notamment une bouche fermée et des lèvres minces, signes de qualité et de dignité ${ }^{36}$, une attitude sereine, mesurée, harmonieuse et très souvent en extension complète.

La situation des personnages est également importante : Michel combat le dragon dans le ciel ou tombé à terre mais n'a jamais les pieds sur le sol. Signe de sa non-appartenance au monde terrestre ${ }^{37}$, c'est aussi une manifestation de sa domination, de sa victoire sur le mal quand il foule aux

32. Sur ce thème : Cassagnes-Brouquet, Sophie, Les Anges et les démons, Rodez, 1993.

33. GARNIER, F., Le Langage de l'image au Moyen Âge, t. 1, Paris, 1982, p. 133.

34. Pastoureau, Michel, Couleurs, images, symboles. Études d'histoire et d'anthropologie, Paris, 1989, p. 86.

35. Ibid., p. 89.

36. GARNIER, F., op. cit., p. 135.

37. Ibid., p. 103. 
pieds l'être diabolique ${ }^{38}$ et les Heures de Boucicaut comme celles de la Bodleian Library en témoignent. Autre signe de sa domination : le fait de tenir le diable par la barbe comme dans les Heures de Montauban ou par les oreilles comme dans les Heures de Pierre II. C'est, en effet, synonyme de mise à mort ${ }^{39}$. Celle-ci est très clairement exprimée dans les Heures de Boucicaut et celles de la Bodleian Library : l'être malfaisant percé de la lance crucifère gît sur le sol, blessé à mort, malgré quelques tentatives de résistance. Il s'agit là d'une illustration stricte du verset 9 du chapitre 12 de l'Apocalypse : "Et ce grand dragon, cet ancien serpent qui est appelé Diable et Satan, qui séduit tout le monde, fut précipité sur la terre et ses anges avec lui."

Cette opposition et cette victoire se traduisent aussi par le biais des couleurs. L'être diabolique est un être nu dont la couleur fait souvent partie de la gamme des tons sombres - comme le noir dans les Heures de Montauban, le vert très sombre des Heures de la Bodleian Library - en témoignage de son appartenance au monde des ténèbres ${ }^{40}$. Dans les Très Riches Heures du duc de Berry, le dragon est vert-jaune, couleur du Diable, signe de perturbation de la norme et de l'ordre établi ${ }^{41}$ dans l'imaginaire médiéval. On observe aussi l'importance du rouge : griffes dans les Heures de Pierre II, langue dans les Heures de Berry, celles de Montauban ou celles du duc breton mais aussi flammes de l'Enfer. Ce sont là des exemples de ce mauvais rouge, indice de cruauté et d'orgueil ${ }^{42}$, associé à partir du XIII ${ }^{\mathrm{e}}$ siècle aux êtres diaboliques ${ }^{43}$. À ces couleurs très significatives, s'ajoutent poils, plumes et écailles qui expriment les défauts du corps et renforcent son caractère inquiétant ${ }^{44}$. Michel est, lui, très souvent vêtu de rouge, de bleu, de jaune rougeoyant, de blanc, toutes ces couleurs étant signes de diverses valeurs et qualités telles la force, le courage, la pureté, la justice, l'éternité, la noblesse, la souveraineté, la loyauté, la sagesse ${ }^{45}$.

Ses vêtements eux-mêmes ont un sens fort. Ils sont faits à partir de textiles riches et soigneusement finis. En témoignent les orfrois qui ornent son manteau dans les Heures de Montauban, les Heures de Boucicaut, les Heures de la Bodleian Library ou les Heures de Pierre II et qui apportent au personnage une gloire encore plus accrue ${ }^{46}$. Dans les Heures à l'usage de Nantes, la doublure du manteau est blanche semée de noir, signe de majesté et de sacralité. Le port des habits est également traité avec attention par l'artiste : l'ampleur, le nombre considérable de plis, le volume caractéri-

38. Ibid., p. 232.

39. Ibid., p. 137.

40. Pastoureau, Michel, Couleurs, images..., op. cit., p. 96.

41. Id., Figures et couleurs. Étude sur la symbolique et la sensibilité médiévale, Paris, 1986, p. 30 .

42. Ibid., p. 40.

43. Id., Couleurs, images..., op. cit., p. 75.

44. Ibid., p. 92.

45. Id., Figures et couleurs..., op. cit., p. 40.

46. Blanc, O., Parades et parures..., op. cit., p. 156. 
sent la tenue du saint personnage ${ }^{47}$. De même, le fait que Michel ait toujours le manteau relevé sur l'épaule a une signification : ce geste dégage la doublure en signe d'autorité ${ }^{48}$.

On ajoutera enfin l'opposition classique entre gauche et droite ${ }^{49}$ : la prééminence de la main droite, du côté droit, de la place à droite, et, inversement, la défaveur, la perversité de tout ce qui se trouve à gauche sont fortement soulignées dans la Bible ${ }^{50}$ et traduites dans l'art du Moyen Âge chrétien.

Ainsi, par divers codes et conventions, l'artiste représentant cette lutte apocalyptique entre Michel et le dragon en fait une image de l'opposition éternelle entre le Bien et le Mal. Cette image, contemporaine du temps du peintre par certains aspects, revêt aussi un sens symbolique et allégorique fort.

\section{Réalisme, symbolique}

Les enluminures de notre corpus présentent un certain réalisme dans la mesure où elles reproduisent les apparences du monde matériel mais elles sont également dotées de contenus qui ne correspondent pas aux données ordinaires de la perception et sont donc porteuses d'une symbolique forte ${ }^{51}$.

Sans tomber dans une foi aveugle dans ce document qu'est l'image, on peut noter cependant quelques aspects réalistes, notamment dans la représentation des bâtiments religieux dans les Très Riches Heures du duc de Berry et celles de Sobieski en particulier ${ }^{52}$.

Dans le premier cas, on distingue nettement les diverses composantes avec de gauche à droite : les Fanils ou magasins de l'abbaye dans l'extrême gauche, l'hôtellerie, la chapelle Saint-Étienne avec ses trois niveaux de baies, la façade du monastère avant l'édification de la tour de l'Abbé et des logis abbatiaux, la tour Sainte-Catherine avec sa fenêtre unique et ses arcatures lombardes en face sud, une construction fortifiée dominant l'ouvrage avancé en contrebas qui servait d'entrée au XIII ${ }^{\mathrm{e}}$ siècle avant la construction du Châtelet, la salle des Gardes avec ses deux baies cintrées surmontées d'un oculus, la tour des Corbins avec ses six étages et sa flèche fantaisiste, une partie de la façade est de la Merveille. Plus haut : le bâtiment de Belle-Chaise puis l'abbatiale avec son chœur à déambulatoire encore en place avec sa chapelle axiale, la croisée du transept dominée par

47. Ibid., p. 68-70.

48. Ibid., p. 100.

49. HERTz, R., "La prééminence de la main droite. Étude sur la polarité religieuse ", Mélanges de sociologie religieuse et de folklore, 1928, p. 84-127.

50. PASTOUREAU, Michel, Couleurs, images..., op. cit., p. 79.

51. GARNIER, F., Le Langage de l'image au Moyen Âge, t. 2, Paris, 1989, p. 35.

52. Pour ces identifications, Sophie BouRDon, Les représentations iconographiques..., op. cit. 
son clocher carré et percé de baies dont la flèche est encadrée par cinq petits pyramidions élancés, le transept sud avec sa façade sud percée d'un très grand oculus et son côté est doté d'une abside semi-circulaire, la nef avec sa structure à bas-côtés, ses murs garnis de baies cintrées et sa balustrade, et enfin les tours édifiées par Robert de Thorigny au XII ${ }^{\mathrm{e}}$ siècle. Dans ce dernier cas, l'artiste a représenté, par souci d'équilibre pour l'ensemble, la tour nord même si elle n'existait déjà plus.

Dans les Heures de Sobieski, malgré une attention très limitée du peintre pour l'assemblage des différents bâtiments entre eux, on peut identifier les lieux grâce à son souci du détail. De gauche à droite : une tourelle quadrangulaire surmontée d'un campanile correspondant dans sa forme à la tour sud de Robert de Thorigny mais s'en détachant par son emplacement, le mur d'enceinte des jardins contournant la tour par derrière, à l'est de la tour un grand bâtiment ressemblant, malgré le problème de position, à la tour de l'Abbé avec ses petites ouvertures carrées, ses deux rangées de baies, ses meurtrières et sa toiture en terrasse, les remparts avec leur chemin de ronde et sa tour qui doit être celle de Sainte-Catherine, la salle des Gardes avec ses baies surmontées d'un oculus, le bâtiment de Belle-Chaise et sa balustrade, le Châtelet avec son front crénelé, le pignon est de la Merveille surmonté d'une croix, la tour des Corbins avec sa toiture pointue et sa section polygonale. L'abbatiale avec un côté ouest rendu étrange par l'enchevêtrement de trois pignons, le transept sud, le clocher avec sa balustrade, sa flèche et ses clochetons, le chœur roman avec sa chapelle axiale, la nef identifiable par ses baies est mal placée par rapport au transept.

Dans ces deux cas, l'artiste s'est déplacé sur les lieux et en a fait une représentation fidèle et précise notamment dans les détails architecturaux. Le but est de donner une image du Mont qui soit comprise du spectateur : ainsi, le réalisme peut être remplacé par du figuratif comme dans les autres enluminures de notre corpus. Dans les Heures de Montauban, on reconnaît ainsi le clocher au centre avec ses clochetons, la nef avec ses baies, une tour qui pourrait être celle de Sainte-Catherine, les deux autres aux toits orangés pouvant être celles de Robert de Thorigny.

Le réalisme est aussi présent par le biais de l'anachronisme de l'iconographie médiévale : ainsi, l'armement dont est pourvu l'archange est celui des contemporains de l'enluminure. L'épée d'estoc des Très Riches Heures du duc de Berry, des Heures dites de Sobieski, des Heures de Pierre II et des Heures de Montauban comme l'armure intégrale présente dans ces deux derniers manuscrits sont celles des chevaliers du Xve siècle. Cet anachronisme se manifeste aussi dans les vêtements des pèlerins, en particulier dans les Heures de Sobieski et celles de Pierre II ${ }^{53}$.

L'enluminure du manuscrit rennais est aussi réaliste dans la mesure où elle représente des faits quasiment contemporains de la réalisation de l'œu-

53. Sur les vêtements au Moyen Âge : BLAnc, O., Parades et parures..., op. cit. 
vre. En effet, les étendards et les oriflammes fleurdelisés aux couleurs bleu et or du roi de France ${ }^{54}$ présents dans ce seul folio et en très grand nombre affirment très fortement la résistance des habitants du Mont aux attaques anglaises dans la première moitié du Xve siècle, notamment après les conquêtes du roi Henri V en 1417 et lors du siège de 1427. Cette opposition franco-anglaise et cette résistance sont renforcées par la représentation des navires anglais dans la baie. En effet, on observe sur le haut des mâts des bannières rouges, couleur des Plantagenêt depuis le début du $\mathrm{XII}^{\mathrm{e}}$ siècle ${ }^{55}$, et dans la barque l'homme à la livrée rouge et or ne peut être qu'un Anglais car dans l'imaginaire des habitants du continent un sujet du roi d'Angleterre est toujours vêtu ainsi ${ }^{56}$.

Autre combat toujours dans cette enluminure : celui de Michel contre l'être diabolique. Il peut être l'illustration de la défaite des forces malfaisantes à la fin du monde ou une représentation de la lutte éternelle du Bien contre le Mal. Dans ce cadre, Michel serait symbole du Christ, le combat une allégorie de la Crucifixion et du baptême ${ }^{57}$. L'assimilation entre la Crucifixion et le combat a été assez simple : comme le Christ a terrassé le serpent par la croix, l'archange transperce le dragon de sa lance ${ }^{58}$. Toujours symbole de la lutte du Bien contre le Mal, le combat archangélique peut être aussi une allégorie du baptême ${ }^{59}$ vu comme le renouvellement, par le catéchumène, de la mort, de l'ensevelissement et de la résurrection du Christ ${ }^{60}$ selon que Paul écrivait aux Romains : " Nous avons donc été ensevelis avec lui dans la mort par le baptême, afin que, comme Christ est ressuscité d'entre les morts par la gloire du Père, de même nous aussi nous marchions en nouveauté de vie ${ }^{61}$."

Il faut cependant conserver à l'esprit que la dévotion envers Michel est surtout celle envers l'archange de la pesée des âmes lors du Jugement dernier et envers le combattant du Mal. Son rôle consiste à remporter la dispute de l'âme : il est alors, comme dans les Heures de Montauban, de Boucicaut, de Sobieski et celles de la Bodleian Library, fréquemment armé d'une lance crucifère quand il combat le démon. L'utilisation de la croix pour combattre l'être diabolique a un sens théologique fort : en dotant l'archange d'une telle arme, l'artiste se conforme au texte de l'Apocalypse : « Et ils l'ont vaincu par le sang de l'Agneau ${ }^{62}$. " C'est armé du même objet qu'il

54. Sur ce thème : Lombard-Jourdan, A., Fleur de lis et oriflamme. Signes célestes du royaume de France, Paris, 1991.

55. PAStoureau, Michel, Figures et couleurs..., op. cit., p. 64.

56. Ibid., p. 65.

57. AvRIL, F., "Interprétations symboliques du combat de saint Michel et du dragon ", Millénaire monastique du Mont-Saint-Michel, t. 3 : Culte de saint Michel et pèlerinages au Mont, Paris, 1971, p. 39-52, p. 40

58. Ibid., p. 45.

59. Ibid., p. 49.

60. Ibid., p. 50 .

61. Épître de Paul aux Romains, VI, 3-4.

62. Apocalypse de Jean, XII, 11. 
intervient dans la pesée représentée dans le manuscrit rennais. Se rejoignent alors les dimensions narrative et symbolique du rôle de l'archange : sa lutte triomphante contre le Mal est l'équivalent, pour chaque individu, de la victoire à vocation universelle du Christ lors de la Crucifixion.

On peut aussi voir dans l'utilisation de la lance crucifère des Heures de Montauban une autre dimension : le sacrifice du Christ au Calvaire serait un argument avancé dans la plaidoirie qui permettrait d'obtenir le salut de l'âme. Dans ce contexte très juridique, le terme adjutorium prend alors un sens fort : l'aide apportée par le saint est un secours officiel, administratif. Toujours dans le même manuscrit et allant dans le même sens : les deux anges qui conduisent les élus au Paradis sont munis d'une croix. Dans ces deux cas, la croix ne sert pas à écarter le mal mais à permettre le salut. Ainsi, si la lutte michaëlienne peut être une allégorie de la Crucifixion, elle transmet quand même l'idée essentielle du christianisme : c'est par le sang du Christ que le diable est vaincu et le salut obtenu pour les hommes. On ajoutera ici une hypothèse concernant le personnage féminin présent derrière l'archange dans la scène du Jugement dernier : il est vêtu d'une robe rouge à bordure dorée caractéristique de la Vierge et destinée à mettre en avant la gloire qu'elle a acquise en partageant la dignité impériale avec son Fils dans les cieux ${ }^{63}$. De plus, ses gestes, son attitude, son regard expriment une profonde bienveillance, quasiment maternelle, envers les âmes destinées au Paradis. Cette vision de Marie comme mère de l'Église est une des conséquences de l'idée de médiation maternelle énoncée par Bernard de Clairvaux dans la première moitié du XII ${ }^{\mathrm{e}}$ siècle ${ }^{64}$. Enfin, sa position et sa situation dans l'enluminure sont intéressantes : le personnage est placé derrière Michel mais n'a pas encore franchi le cadre séparant le lieu du Jugement de l'Au-delà bienheureux. On peut ainsi penser que ce personnage a un rôle à jouer dans l'accession au salut et cette hypothèse est confortée par les écrits de saint Bonaventure : il a développé le thème de Marie coopérante à l'œuvre rédemptrice du Christ en employant également le terme adjutorium ${ }^{65}$. L'intervention très officielle de ce personnage qui présente les élus à l'entrée de la cité céleste est donc considérée comme nécessaire voire indispensable pour accéder au Paradis. Il est ainsi fort probable que ce personnage féminin et apparemment ailé soit la Vierge. On observera alors avec quel soin est transcrit en image le dogme catholique du salut obtenu par le sang du Christ certes, mais aussi grâce à l'aide de la Vierge et des saints.

Michel, archange du Jugement, peut aussi apparaître en simple combattant armé d'une épée comme dans l'ensemble de notre corpus : il apparaît ainsi comme le " chevalier céleste au service de l'infinie Miséricorde ${ }^{66}$ "

63. Delumeau, Jean, Que reste-t-il..., op. cit., p. 141.

64. Roux, H., "Marie ", Dictionnaire de théologie chrétienne, Paris, 1998, p. 538-543, p. 542. 65. Ibid.

66. FouRnEe, J., "L'archange de la mort et du Jugement ", Millénaire monastique..., op. cit., p. 65-91, p. 87. Il est présenté ainsi dans le Livre de Daniel XII, 1 : «En ce temps-là se lèvera Michel, le grand chef, celui qui tient bon en faveur des fils de ton peuple. " 
plus que comme l'archange intervenant pour sauver la femme devant enfanter mentionnée au chapitre 12 de l'Apocalypse. Le narratif s'efface devant le symbolique : Michel combat pour tous et pour le salut des âmes qu'il conduit ensuite au Paradis. On adopte alors la lecture " dans le ciel, c'està-dire pour l'Église ${ }^{67}$ " de Césaire d'Arles : Michel combat non pas dans le ciel mais dans le but de sauver l'Église dont l'existence même est signe de la défaite diabolique.

Cette double fonction de juge et de combattant s'exprime par la tenue : l'archange juge est vêtu d'une grande robe blanche comme dans le Polyptyque du Jugement dernier de Rogier Van der Weyden et le Michel en armure combattant l'être malfaisant est très fréquent aussi, notre corpus en témoigne. On observe cependant que les deux iconographies peuvent se mêler : dans les Heures dites de Sobieski et dans les Heures de Montauban, Michel combat uniquement revêtu de sa tenue immaculée et inversement, le peintre des Heures de Montauban le représente soutenant la balance, protégé par une armure et armé d'une lance crucifère lors du Jugement. Ainsi, les deux fonctions tendent à être confondues et rendues indissociables : toute son action est lutte et tous ses combats sont intervention salvatrice.

Dans les Heures dites de Sobieski, on observe que la marche vers la Jérusalem céleste sous la protection de l'archange commence par le secours apporté aux pèlerins se rendant au sanctuaire normand. Le pèlerinage apparaît alors comme une étape de ce chemin vers l'Au-delà ou une allégorie de la marche du fidèle vers la cité céleste. En effet, les dangers de la baie, la réputation du lieu saint, la perception du combat comme une image du baptême et de la Crucifixion pourraient conduire à voir le MontSaint-Michel comme une image de l'Au-delà ${ }^{68}$.

Les enluminures du livre d'heures de Pierre II et du livre de la Bodleian Library associent également combat archangélique et pèlerinage : dans le premier cas, le cadre consacré à la lutte semble primer sur celui où est représenté le Mont et les pèlerins et dans le second, le sanctuaire et les fidèles occupent l'arrière-plan uniquement. Dans ces deux cas, la victoire de Michel justifie le pèlerinage mais le rend possible également. Derrière des scènes narratives, l'allégorique et le symbolique se rejoignent pour faire du sanctuaire une image du Paradis, du pèlerinage et de ses aléas, le chemin menant dans l'Au-delà, le tout étant possible par l'intervention salvatrice de Michel. La présence du paon, symbole d'éternité et d'immortalité, juste au-dessus du Mont dans les heures du duc breton renforcerait cette hypothèse.

L'enluminure des Heures de Montauban est très intéressante car elle présente un cycle et nous permettra de conclure : le Mont-Saint-Michel est l'élément central et l'archange en assure la défense face aux attaques ou incursions diaboliques; à droite, Michel intervient pour sauver une âme;

67. Cesaire D’Arles, dans Migne, Patrologia latina, t. 100, col. 1154.

68. Bourdon, Sophie, Les représentations iconographiques..., op. cit., p. 270. 
en bas à droite, il remporte la victoire sur le diable rendant ainsi possible le salut; en bas au centre, Michel intervient partialement dans le Jugement de l'âme et en bas à gauche, l'Église triomphante est conduite dans la Jérusalem céleste. L'association est faite : l'archange honoré au Mont-SaintMichel protège son sanctuaire et veille au salut éternel des fidèles assurés de parvenir, par son aide, au Paradis. La colombe, à gauche du Mont, est la forme corporelle sous laquelle se manifeste l'esprit de Dieu ${ }^{69}$ : ainsi, le sanctuaire protégé de l'archange est le lieu où réside l'esprit de Dieu, ce qui l'assimile au royaume divin malgré sa réalité terrestre, ce qui donne également à la résistance française face aux Anglais une dimension religieuse. Ainsi, la signification de cette enluminure est clairement politisée : en commandant ce livre d'heures dans les années 1430-1440, Jean de Montauban affirme le pouvoir du roi de France ${ }^{70}$, sa résistance aux Anglais assimilés au Diable mais aussi la protection spéciale qu'assure Michel au royaume dans sa totalité et à ce lieu saint en particulier.

\section{RESUME}

Basée sur une enluminure inédite extraite d'un manuscrit breton, cette étude est ensuite élargie à l'ensemble des miniatures associant l'archange Michel au sanctuaire normand. Il s'agit, après une approche descriptive, d'analyser ces représentations pour en dégager les significations symboliques tant religieuses que politiques. Le Mont-Saint-Michel apparaît alors comme un lieu saint, témoin de la protection spéciale qu'accorde l'archange à son sanctuaire et au royaume de France en général.

\section{ABSTRACT}

Grounded on an unpublished illumination taken from a Breton manuscript, this research has then been broadened to the whole set of miniatures which associate Archangel Michael with the Norman sanctuary. After having led an explanatory approach, it is a matter of analysing these representations in order to bring out their religious as well as their political symbolical meanings. Mount Saint Michael then appears as a holy place which testifies of the special protection the archangel attached both to his sanctuary and to the kingdom of France in general.

69. Évangile de Matthieu, III, 13-17.

70. Sur les liens entre peintres et politique : CASSAGNES-BROUQUET, Sophie, « Les peintres et la politique : l'exemple de la Bourgogne des ducs Valois ", JOUBERT, F. (dir.), L'artiste et le commanditaire aux derniers siècles du Moyen Âge (XIII'-XVI siècles), Paris, 2001, p. 83-97. 